\title{
PUNCHING FORGINGS «CROSSPIECE» FROM BALL BLANKS
}

Filippova M.V., Cand. Sci. (Eng.) Assist. Professor

(filippova_mv@mail.ru)

Peretayt'ko V.N., Dr. Sci. (Eng.), Professor

Fedorov A.A., Cand. Sci. (Eng.), Assist. Professor

Klimov A.S., Posgraduate

Siberian State Industrial University (42, Kirova str., Novokuznetsk, Kemerovo Region, 654007, Russia)

Abstract. The new extruding technology of forging of «crosspiece» type from a ball blank subjected to two-sided force has been developed. The study of the stress-strain state of the metal has been conducted. The pilot batch of high quality forgings of «crosspiece» type for cardan shaft of the vehicle has been produced.

Keywords: stamping, extrusion, ball storage, stress, strain, average normal stress, quality forgings.

\section{REFERENCES}

1. Eduardov M.S. Shtampovka v zakrytykh shtampakh [Closed-die forging]. Moscow: Mashinostroenie, 1971. 240 p. (In Russ.).

2. Oskolkov A.I., Maksimov A.A., Poksevatkin M.I., Peretyat'ko V.N. etc. Sposob shtampovki pokovok tipa krestovin [Forming method of forgings of Crossings type]. Patent RF no. 2165329. Byul. Izobretenii, no. 4, 2001. (In Russ.).

3. Filippova M.V., Bakhaev A.V., Peretyat'ko V.N., Fedorov A.A. Ball billet die forming in formed hot modeling. Izvestiya VUZov. Cher- naya metallurgiya $=$ Izvestiya - Ferrous Metallurgy. 2011, no. 10, pp. 24-27. (In Russ.).

4. Peretyat'ko V.N., Bakhaev A.V., Filippova M.V. Modeling of axially symmetric forging die stamp process. Izvestiya VUZov. Chernaya metallurgiya $=$ Izvestiya - Ferrous Metallurgy. 2010, no. 4, pp. 27-29. (In Russ.).

5. Peretyat'ko V.N., Bakhaev A.V., Filippova M.V., Vakhman S.A. Stamping axisymmetric forgings. Steel in Translation. 2009. Vol. 39, no. 4, pp. 300-301.

6. Tselikov A.I., Barbarin M.V., Vasil'chikovM.V. etc. Spetsial'nye prokatnye stany [Special mills]. Moscow: Metallurgiya, 1971. 336 p. (In Russ.).

7. Peretyat'ko V.N., Filippova M.V., Klimov A.S. etc. Ball blank. Zagotovitel'noe proizvodstvo v mashinostroenii. 2012, no. 3, pp. 17-19. (In Russ.).

8. Peretyat'ko V.N., Fedorov A.A. Shtamp dlya goryachei shtampovki pokovok tipa krestovina iz sharovoi zagotovki [Stamp for hot stamping forgings of crossings type from ball blank]. Patent RF na poleznuju model' RF, MPK V21K. Byul. Izobretii, no. 1, 2010. (In Russ.).

9. Ovchinnikov A.G. Osnovy teorii shtampovki vydavlivaniem na pressakh [The theory fundamentals of extruding presses]. Moscow: Mashinostroenie, 1983. 197 p.

10. Peretyat'ko V.N., Bakhaev A.V., Filippova M.V., Vakhman S.A. Hot forming of axially symmetric forgings. Izvestiya VUZov. Chernaya metallurgiya $=$ Izvestiya - Ferrous Metallurgy. 2009, no. 4, pp. 21-22. (In Russ.).

\section{ИЗУЧЕНИЕ ОСОБЕННОСТЕЙ ПРИМЕНЕНИЯ ВИХРЕВЫХ ТЕЧЕНИЙ ДЛЯ НАНЕСЕНИЯ ОГНЕУПОРНЫХ ПОКРЫТИЙ НА ФУТЕРОВКУ КОНВЕРТЕРОВ*}

\author{
Фейлер С.В. ${ }^{1}$, к.т.н., доиент (feyler@rdtc.ru) \\ Протопопов $\boldsymbol{E . B .}{ }^{1}$, д.т.н., профессор \\ Чернятевич A.Г. ${ }^{2}$, д.т.н., профессор \\ Калиногорский А.Н.' ${ }^{1}$, аспирант \\ Багрянцев В.И. ${ }^{1}$, к.т.н.
}

\footnotetext{
${ }^{1}$ Сибирский государственный индустриальный университет (654007, Россия, Новокузнецк, Кемеровской обл., ул. Кирова, 42)

2 Днепродзержинский государственный технический университет

(Украина, Днепродзержинск, ул. Днепростроевская, д. 2)
}

Аннотация. С использованием методов физического моделирования изучены режимы истечения вихревых газовых потоков из сопел различного диаметра при различном расходе газа.

Ключевые слова: конвертер, стойкость футеровки, раздувка шлака, шлаковый гарнисаж, вихревые потоки.

Эффективная реализация технологии нанесения шлакового гарнисажа на футеровку кислородных конвертеров

* Работа выполнена в СибГИУ по государственному заданию Минобрнауки РФ, проект 2556. предусматривает использование специальных дутьевых устройств и определенного дутьевого режима операции $[1,2]$. Новая теоретическая и экспериментальная информация в этом направлении позволяет в промышленных условиях принимать более обоснованные технические решения. 
Одним из перспективных направлений оптимизации работы специальных дутьевых устройств для нанесения шлакового гарнисажа является использование в фурмах элементов конструкции вихревых аппаратов для получения вихревых газовых струй [3, 4], что позволяет обеспечить максимально возможное разбрызгивание расплава и равномерное нанесение гарнисажа на верхние горизонты футеровки конвертера.

Как известно, механизм взаимодействия газовых струй со шлаковым расплавом с высокой степенью достоверности можно изучать с использованием методов физического моделирования процессов. В настоящей работе особенности гидродинамики шлаковой ванны изучали методами низкотемпературного моделирования с использованием усовершенствованной установки [5] на основе прозрачной плексигласовой модели конвертера (рис. 1), профиль и размеры рабочего пространства которой выполнены в масштабе 1:20 по отношению к 350-т агрегатам ОАО «ЕВРАЗ Объединенный Западно-Сибирский металлургический комбинат» (ОАО «ЕВРАЗ ЗСМК»). Применяемые установка и методика исследований позволяют изучать особенности раздувки крахмального клейстера, моделирующего шлаковый расплав, воздушными или воздушно-порошковыми газовыми струями различной структуры при
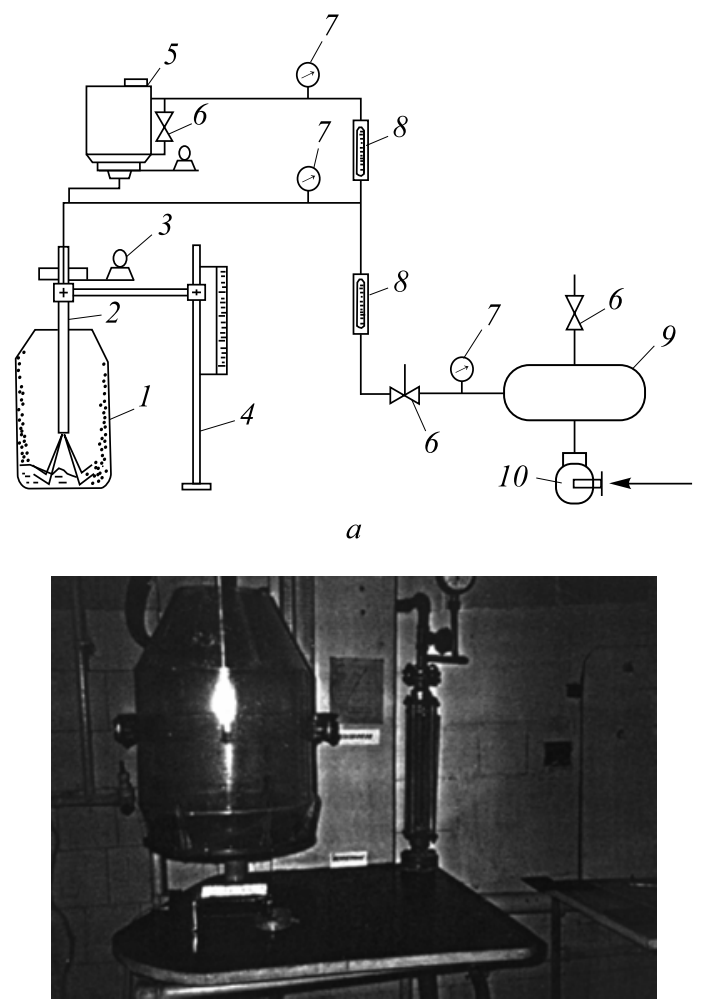

6

Рис. 1. Схема установки физического моделирования процесса нанесения шлакового гарнисажа $(a)$ и фотография плексигласовой модели конвертера (б):

1 - плексигласовая модель конвертера; 2 - фурма; 3 - привод вращения фурмы; 4 - устройство крепления и перемещения фурмы;

5 - роторный флюсопитатель; 6 - вентиль; 7 - манометр; 8 - ротаметр; 9 - ресивер; 10 - компрессор фиксировании процесса цифровой видеокамерой в различных ракурсах.

Использование результатов моделирования в реальном технологическом процессе обеспечивается соблюдением равенства следующих критериев:

$$
\begin{aligned}
& i_{\mathrm{\Gamma}} \cos \alpha \frac{n}{m_{\text {ж }} g}=\text { idem; } i_{\mathrm{r} . \mathrm{I}} \cos \alpha \frac{n}{m_{\text {ж }} g}=\text { idem; } \\
& \frac{H_{\phi}}{d_{\text {ц }}}=i d e m ; \frac{H_{\phi}}{H_{\text {я }}}=i d e m ; \frac{d_{\mathrm{o}}}{D_{\text {ц }}}=i d e m,
\end{aligned}
$$

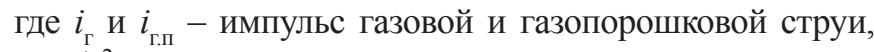
кг $\mathrm{M} / \mathrm{c}^{2} ; \alpha-$ угол наклона сопел к вертикальной оси фурмы, град; $n$ - количество сопел в наконечнике, шт.; $m_{\text {ж }}=K_{\mathrm{\kappa}} \rho_{\text {ж }} F_{\text {ж }} h_{\text {ж }}$ - масса жидкого шлака, кг; $K_{\mathrm{\kappa}}-$ коэффициент, учитывающий форму днища конвертера; $\rho_{ж}-$ плотность шлакового расплава, кг $/ \mathrm{M}^{3} ; F_{\text {ж }}$ - площадь поверхности шлаковой ванны, $\mathrm{m}^{2} ; h_{\text {ж }}$ - глубина жидкой ванны в спокойном состоянии, м; $H_{\phi}$ - высота нахождения фурмы, м; $d_{\text {щ }}$ - диаметр сопла, $\mathrm{m} ; H_{\text {я }}$ высота размещения боковых сопел на стволе фурмы относительно торца наконечника, м; $d_{\text {o }}$ - диаметр окружности расположения осей выходных сечений сопел на торце наконечника, м; $D_{\text {ц }}$ - диаметр цилиндрической части конвертера, м.

Как установлено в ходе проведенных экспериментов, при обычном варианте раздувки клейстера газовыми струями наблюдаются $[5,6]$ два основных гидрогазодинамических режима взаимодействия (рис. 2) при глубине ванны, соответствующей количеству оставляемого конечного шлака (примерно $10-12 \%$ от массы жидкой стали).

При первом режиме взаимодействия раздувка шлака реализуется при внедрении газовых струй на глубину, не превышающую глубину шлаковой ванны, при втором режиме происходит пробой струями слоя шлакового расплава. Второй режим взаимодействия многоструйного газового дутья со шлаковой ванной является основным в практике гарантированного нанесения шлакового гарнисажа по всей высоте футеровки.

При реализации первого режима взаимодействия под действием отраженного потока газа у границ каждого кратера формируются гребни волн, которые перемещаются по поверхности кратера в радиальном направлении. При этом на периферии образуется своеобразный вал (возвышение) над уровнем жидкости, с вершины которого отрываются, прежде всего, крупные всплески и брызги клейстера с преимущественным направленным выносом более мелких капель по траектории отраженного потока. По мере увеличения диаметра эллипсовидного кратера по большой оси и при приближении внешней границы вала к поверхности футеровки конвертера (что происходит в случае увеличения значений $\left.Q_{\Gamma}, H_{\phi}, \alpha\right)$ крупные всплески и брызги жидкости все в большем количестве выносятся на стенки конвертера, создавая своеобразный пленочный слой. При этом траектория направленного выноса мелких капель изме- 


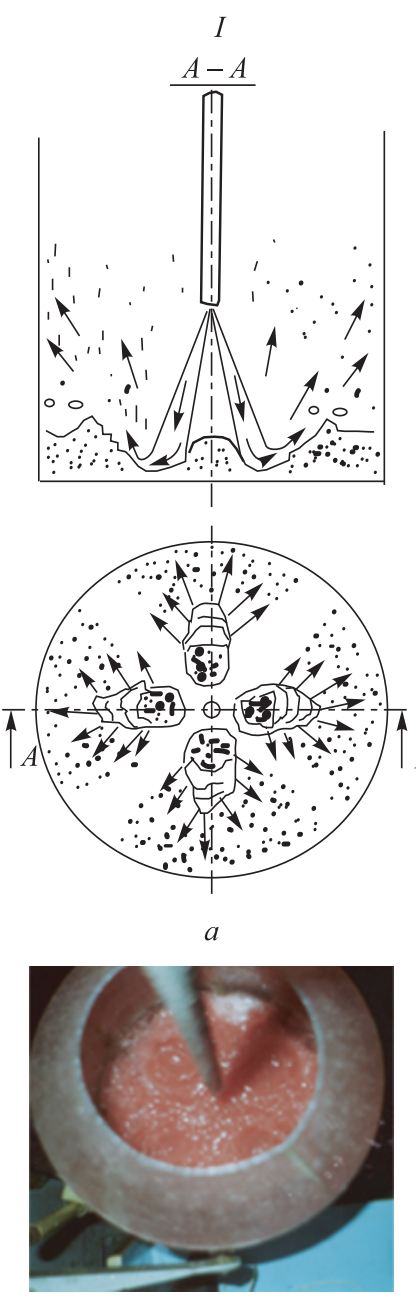

6

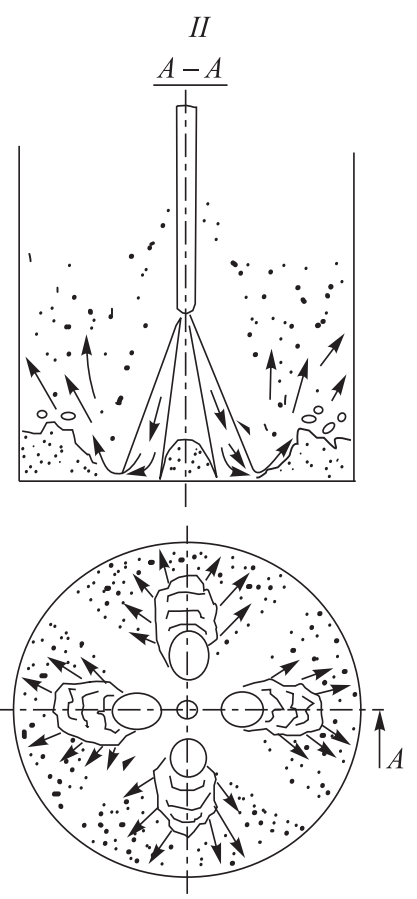

$a$

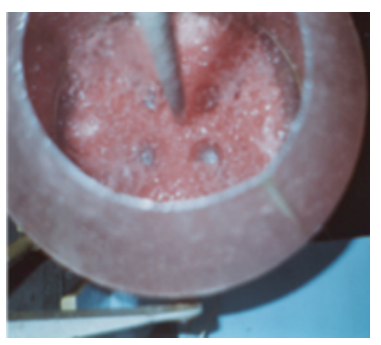

6
Рис. 2. Схемы (a) и фотографии (б) режима $I(I)$ и режима $I I(I I)$ взаимодействия воздушных струй с крахмальным клейстером при использовании четырехсоплового наконечника

няется в сторону снижения уровня (высоты) попадания капель на футеровку, то есть возможной высоты нанесения шлакового гарнисажа. Необходимо отметить, что в этом случае, как правило, образующиеся кратеры стабилизированы в шлаковой ванне относительно вертикальной оси фурмы. Разрушение всплесков с направленным выносом брызг на стенки конвертера обусловлено, прежде всего, подрезанием гребня волны на краю вала отраженным от основания кратера газовым потоком. Очевидно, что значительно меньший объем брызговыноса при таком режиме связан с разрушением гребня по поверхности кратера волн. При этом зона максимального брызговыноса из каждого обособленного кратера (при визуализации в плане (рис. 2, a)) сосредоточена на стороне воздействия отраженного потока газа на жидкость, то есть в ближнем к стенкам конвертера полуобъеме эллипсовидного кратера.

Второй режим взаимодействия многоструйного дутья с жидкой ванной малой высоты (рис. 2, б) характеризуется картиной, когда в рассматриваемом диапазоне изменения величин $Q_{\Gamma}, H_{\phi}, \alpha$ происходит пробой газовыми струями столба жидкости с образованием мощного отраженного от днища обратного газожидкостного потока. При этом формируются обособленные или соприкасающиеся кратеры эллипсовидной формы, что является определяющим и основным фактором в обеспечении эффективности раздувки шлака с целью нанесения гарнисажа на футеровку конвертера. В данном случае диаметр основания обособленного кратера на зеркале ванны (рис. 2, б) практически совпадает с диаметром вдуваемой наклонной струи на этом уровне, а обратный газовый поток вызывает перемещение жидкости по поверхности кратера с развитием брызговыноса, с возникновением и разрушением крупных всплесков, особенно на возвышающейся периферийной части кратера. На периферии кратера (в случае приближения последнего к футеровке) наблюдается разрушение гребня как катящейся по поверхности волны, так и отраженной от стенки агрегата обратной волны, распространяющейся по поверхности ванны.

При оптимальных значениях $H_{\phi}$ и $h_{\text {ж }}$ формируются вытянутые в направлении большей оси эллипсовидные кратеры с периодически «захлопывающимися» жидкостью периферийными участками (рис. 2).

В результате создающегося препятствия на пути движения отраженного от днища газожидкостного потока со стороны объемов шлаковой ванны, образующих своеобразную жидкую оболочку («крышу») над периферийной областью кратера, после эффекта «захлопывания» последней происходит разрушение оболочки с взрывным характером брызговыноса шлака на футеровку конвертера по всей высоте рабочего пространства. Снижение высоты фурмы ниже оптимальных значений при фиксированном расходе дутья ведет к уменьшению диаметра кратера по большой оси, форма кратера на поверхности ванны приближается к окружности, происходит снижение интенсивности направленного на футеровку агрегата брызговыноса и увеличение его по стволу фурмы и вертикальной оси конвертера за пределы рабочего пространства. Чрезмерный подъем фурмы над ванной приводит к приближению внешних границ кратеров к футеровке. При этом наблюдается омывание волнами и крупными всплесками жидкости футеровки, а высота брызговыноса уменьшается.

Возможным вариантом оптимизации раздувки шлакового расплава по второму режиму является использование специальной вихревой фурмы. Принцип работы такой фурмы заключается в создании вихревого газового потока в специальной камере [7] и его характерном истечении из центрального выходного сопла.

Для определения аэродинамических особенностей поведения вихревого потока были исследованы поля скоростей закрученной струи на выходе из сопла фурмы. Исследования выполнены с использованием лазерного доплеровского измерителя скорости, при этом диаметр выходного сопла варьировали в пределах от 

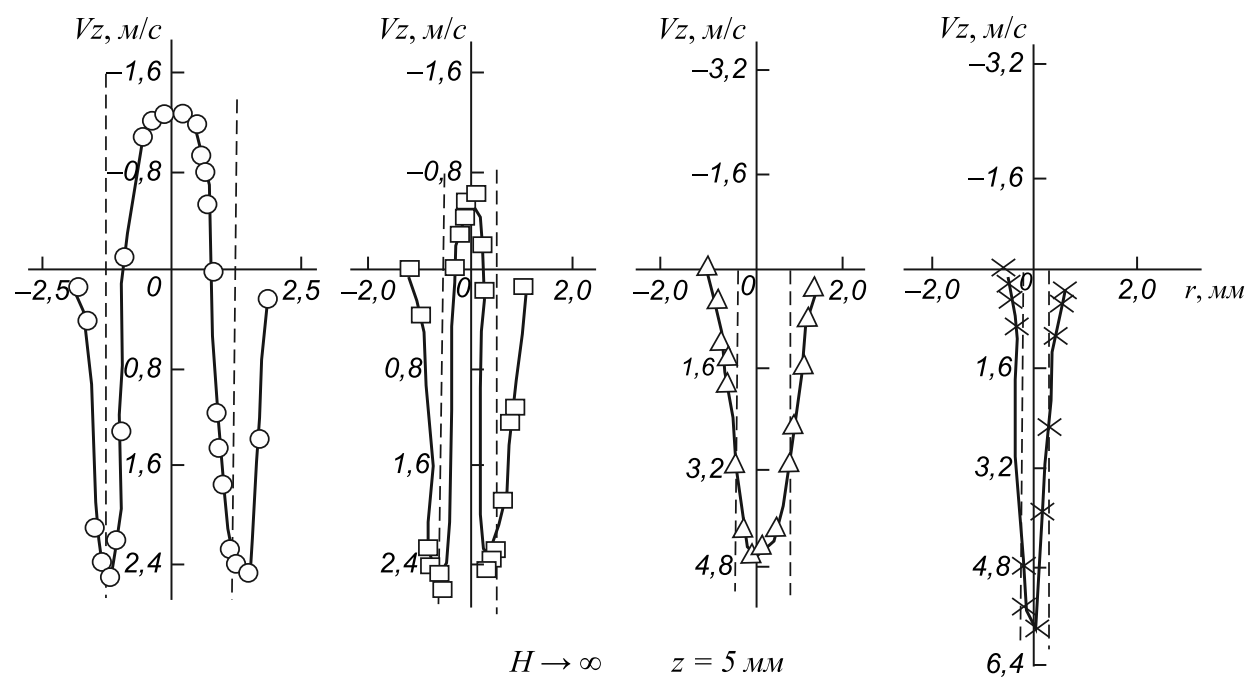

Рис. 3. Распределение осевой компоненты скорости при различных диаметрах $(d)$ выходного сопла и различных расходах $\left(Q_{\mathrm{p}}\right)$ воздуха: O- $Q_{\mathrm{p}}=29,0$ г/ч, $d=2,5 \mathrm{мм} ; \square-Q_{\mathrm{p}}=9,1$ г/ч, $d=1,0 \mathrm{мм} ; \Delta-Q_{\mathrm{p}}=18,8$ г/ч, $d=1,0 \mathrm{мм} ; \times-Q_{\mathrm{p}}=9,1$ г $/ \mathrm{\varphi}, d=0,5$ мм

0,5 до 2,5 мм. Распределение осевой компоненты скорости на выходе из сопла вихревой фурмы представлено на рис. 3.

Как видно, профиль осевой компоненты скорости зависит от диаметра выходного сечения сопла и расхода воздуха. При диаметре 2,5 мм фиксируется зона интенсивного возвратного потока, при диаметре 0,5 мм профиль скорости напоминает профиль обычной струи, а при диаметре сопла 1,0 мм появляющееся возвратное течение возникает только при определенном расходе газа. Фактические измерения выполнены на расстоянии 5 мм от среза сопла (на рис. 3 штриховой линией показаны геометрические границы выходного сечения сопла).

В сечении струи профиль тангенциальной скорости сохраняется при закрутке потока, а основной характеристикой является положение максимума скорости. При интенсивном закручивании потока максимум скорости смещается к пограничному слою, а при незначительной закрутке максимум приближается к оси распространения газовой струи. Следует предполагать, что смещение вектора тангенциальной скорости при распространении газовой струи обусловлено трением с окружающей средой при более турбулентном течении потока. Профиль интенсивно закрученного потока, как зафиксировано видеосъемкой, приближается к профилю коаксиальной струи, а слабо закрученного потока к профилю обычной струи. В зависимости от высоты расположения фурмы в случае создания интенсивно закрученного потока можно также реализовать два характерных вида взаимодействия вихревой струи и шлакового расплава: вариант 1 - над поверхностью расплава создается вихрь, который втягивает шлак и отбрасывает его в сторону стенок конвертера (рис. $4, a$ ); вариант 2 струя в условиях «пробоя» оттесняет жидкость на периферию и дробление происходит на некотором удалении от фурмы (рис. 4, б).
Размер капель шлакового расплава в данном случае, очевидно, определяется двумя основными параметрами - затратами энергии на образование новой поверхности и преодолением сил вязкого трения при деформации капли. Часть энергии струи при этом затрачивается на разгон частиц шлака, а значительная

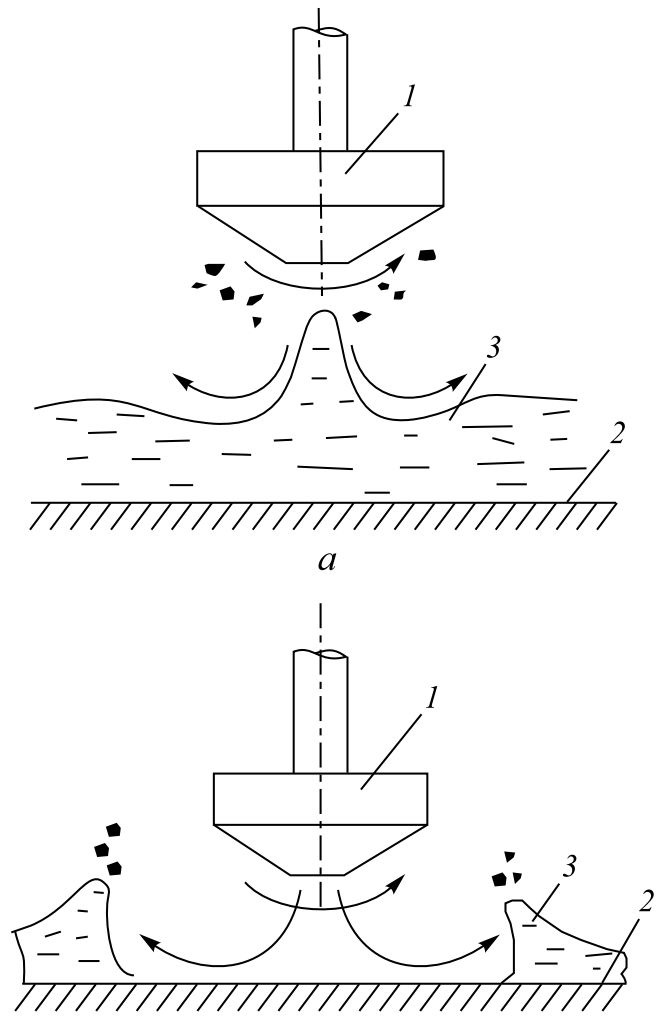

6

Рис. 4. Раздувка шлакового расплава с использованием вихревой фурмы с реализацией режимов взаимодействия газового потока со шлаковым расплавом с зоной пониженного давления $(a)$ и оттеснением расплава от днища (б):

1 - фурма с вихревой камерой; 2 - футеровка днища конвертера; 3 - шлаковый расплав 
часть сохраняется в виде кинетической энергии вихревого газового потока.

Размер граничной области раздела трех фаз (газовый поток, шлаковый расплав, футеровка днища конвертера) зависит от степени закрутки потока и расхода газа. При реализации второго варианта по границе жидкой фазы образуется интенсивный отраженный двухфазный поток, который уносится в направлении стенок конвертера. Физически механизм дробления расплава на капли в обоих случаях аналогичен и описывается подобными соотношениями, но в количественном отношении разница может быть существенной.

В случае реализации взаимодействия по варианту 1 к центральной части шлаковой ванны поступает расплав, который затягивается в вихрь и распыляется по внутреннему объему конвертера, при этом основной процесс реализуется в центре вихря. При реализации второго варианта по окружности возникает своеобразная кольцевая зона двухфазного потока, имеющего меньшую плотность, которая в виде пены легко выносится потоком газа на стенки конвертера.

При использовании положений теории подобия можно определить режим течения, и, соответственно, спрогнозировать количество мельчайших частиц, не участвующих в формировании гарнисажа и уносимых отходящим потоком в газоотводящий тракт. Очевидно, диаметр капель, отрывающихся от поверхности шлакового расплава на границе области раздела трех фаз, должен быть значительно больше, потому что скорость газа на данном участке ниже, чем на границе образующегося вихря, а масса присоединенной жидкости значительно больше. Причем механизм взаимодействия газового потока и шлакового расплава будет аналогичен, но при реализации второго варианта размер капель будет меньше из-за снижения скорости и увеличения массы жидкости, вовлеченной в процесс дробления.

При исследовании режимов взаимодействия вихревого газового потока с шлаковым расплавом (рис. 4, a) установлено, что положение зоны подъема расплава газовым вихрем определяется выходным сечением сопла фурмы. Согласно экспериментальным данным $[8,9]$ уровень турбулентных пульсаций на выходе из вихревой камеры может достигать очень больших величин (вплоть до 100 \%). Предположим, что при распылении капиллярные и вязкие силы имеют один порядок величины, а в области взаимодействия при распылении энергия восполняется за счет притока газа. Поток кинетической энергии газа $\rho_{\Gamma} \Delta u^{2} u S_{\phi} \tau^{2 / 3}$ (где

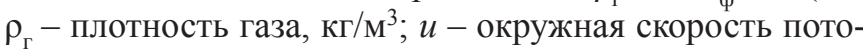
ка, м/с; $\Delta u$ - разность скоростей частицы и пульсаций газовой среды, м/c; $S_{\phi}$ - площадь истечения газовой струи по эффективному диаметру вихря (максимуму тангенциальной скорости), $\mathbf{m}^{2} ; \tau=Q_{\text {ж }} / Q_{\text {г }}$ - отношение объемных расходов газа и жидкой фазы; $\tau^{2 / 3}$ - доля сечения, занятая каплями) расходуется на преодоление сил вязкого трения при деформации капли $\pi \mu_{\Gamma} \Delta u n_{\text {ч }} D^{2}$ (где $\mu_{\Gamma}-$ коэффициент динамической вязкости газа, Па·с; $n_{\text {ч }}$ - число частиц, поступающих в область дробления в единицу времени; $D$ - размер частиц, м) и образование дополнительной поверхности раздела фаз $\pi \sigma n_{\mathrm{q}} D^{2}$ (где $\sigma-$ коэффициент поверхностного натяжения, Н/м). Приравняв соотношения, получим

$$
\rho_{\Gamma} \Delta u^{2} u S_{\phi} \tau^{2 / 3} \approx \pi \mu_{\Gamma} \Delta u n_{\mathrm{u}} D^{2}+\pi \sigma n_{\mathrm{u}} D^{2},
$$

где $\rho_{г} \Delta u^{2}-$ аналог кинетической энергии пульсаций газовой среды, кг/(м· $\left.{ }^{2}\right)$.

Число $n_{\text {ч }}$ определяется по выражению

$$
n_{\text {ч }}=6 \frac{G_{\text {ж }}}{\pi \rho_{\text {ж }} D^{3}} ; \frac{G_{\text {ж }}}{\rho_{\text {ж }}}=Q_{\text {ж }} ; Q_{\Gamma}=u S_{\phi},
$$

где $G_{\text {ж }}$ - массовый расход частиц, кг/с; $\rho_{\text {ж }}-$ плотность шлака, кг/ $\mathbf{M}^{3}$.

Тогда диаметр капель можно оценить по соотношению

$$
D \approx 6 \frac{\tau^{1 / 3}}{\rho_{\Gamma} \Delta u^{2}}\left(\sigma+\mu_{2} \Delta u\right) .
$$

Если левую и правую части полученного соотношения разделить на эффективный диаметр поднятого столба шлака, то получается следующее критериальное уравнение:

$$
\frac{D}{d}=6 \tau^{1 / 3}\left(\mathrm{We}^{-1}+\mathrm{Re}^{-1} \frac{\mu}{\mu_{\Gamma}}\right)
$$

где $\mathrm{We}=\rho_{\Gamma} \Delta u^{2} \frac{d}{\sigma}-$ число Вебера; $\operatorname{Re}=\frac{\rho \Delta u d}{\mu_{г}}-$ число Рейнольдса; $d$ - эффективный диаметр столба шлака, м.

При большей вязкости шлака второй член в выражении (6) намного больше первого, и данное соотношение значительно упрощается.

Физические процессы при диспергировании как в центральной зоне, так и на границе зоны взаимодействия газовой струи с шлаковым расплавом одинаковы, в связи с этим размер капель можно оценить из следующих соображений. В соотношении (6) характерным диаметром является размер граничной области раздела трех фаз, поэтому вводим поправочный коэффициент $K=D / d$. Тангенциальная скорость снижается, исходя из типичного распределения $1 / r$ (это также является отношением диаметров), то есть поправка будет определяться как $(D / d)^{2}$. Пульсационная составляющая взаимодействия также будет снижаться в этих пределах, поэтому при раздувке по периферии возможно увеличение диаметра частиц шлака.

Как известно, вязкость шлакового расплава намного выше вязкости металла, а значение коэффициента поверхностного натяжения невелико, поэтому при оценке размеров частиц можно не учитывать влияние 
числа Вебера. На диаметр частиц, отрывающихся от шлакового расплава, накладывается следующее ограничение: в данном месте образуется двухфазный поток частиц шлака и газа, который выходит из воронки со скоростью, не превышающей скорости звука.

Bbводы. Методами физического моделирования процессов исследованы режимы взаимодействия газового потока с шлаковым расплавом при реализации технологии раздувки шлака на футеровку конвертера при различных конструкциях дутьевых устройств, размерах выходных сопел и расходах газа. Установлена возможность и определены характерные условия для создания оптимального режима раздувки шлака вихревым газовым потоком.

\section{БИБЛИОГРАФИЧЕСКИЙ СПИСОК}

1. Мокринский А.В., Лаврик А.Н., Соколов В.В. и др. // Новые огнеупоры. 2004. № 8. С. $16-18$.
2. Пат. 2128714 РФ. Способ нанесения шлакового гарнисажа на футеровку конвертера / Айзатулов Р.С., Протопопов Е.В., Соколов В.В. и др. Заявл. 24.09.1997. Опубл. 10.04.1999.

3. Bagryantsev V.I., Nikolaev A.L., Poltoratskii L.M., Chevalkov A.V. // Steel in Translation. 1988. Vol. 18. № 2. P. 59 - 61.

4. Bagryantsev V.I., Nikolaev A.L., Pavlenko Z.Ya. // Refractories and Industrial Ceramics. 1988. Vol. 29. № 5-6. Р. 387 - 391.

5. Протопопов Е.В., Калимуллин Р.Ф., Чернятевич А.Г. и др. // Изв. вуз. Черная металлургия. 2012. № 10. С. 38 - 43.

6. Калимуллин Р.Ф., Протопопов Е.В., Чернятевич А.Г., Жибинова И.А. // Вестник СибГИУ. 2013. № 3. С. 9 - 13.

7. Гольдштик М.А. Вихревые потоки. - Новосибирск: Наука, 1981. - $367 \mathrm{c}$.

8. Гупта А., Лилли Д., Сайред Н. Закрученные потоки. - М.: Мир, 1987. - $588 \mathrm{c}$.

9. Кутателадзе С.С., Волчков Э.П., Терехов В.И. Аэродинамика и тепломассообмен в ограниченных вихревых потоках. - Новосибирск: АН СССР, 1987. - 282 с.

(C) 2014 г. Фейлер C.B., Протопопов E.B., Чернятевич А.Г., Калиногорский А.Н., Багрянцев В.И. Поступила 27 июня 2014 г.

Izvestiya VUZov. Chernaya Metallurgiya = Izvestiya - Ferrous Metallurgy. 2014. No. 8. Vol. 57, pp. 28-33.

\section{ANALYSIS OF APPLICATION OF VORTEX MOTION FOR FIRE-RESISTANT COATINGS ON THE VESSEL LINING}

Feiler S.V. ${ }^{1}$, Cand. Sci. (Eng.), Assist. Professor

(feyler@rdtc.ru)

Protopopov E.V. ${ }^{1}$, Dr. Sci. (Eng.), Professor

Chernyatevich A.G. ${ }^{2}$, Dr. Sci. (Eng.), Professor

Kalinogorskii A.N. ${ }^{1}$, Postgraduate

Bagryantsev V.I. ${ }^{1}$, Cand. Sci. (Eng.)

${ }^{1}$ Siberian State Industrial University (42, Kirova str., Novokuznetsk, Kemerovo Region, 654007, Russia)

${ }^{2}$ Dneprodzerzhinsk State Technical University (2, Dneprostroevs-

kaya str., Dneprodzerzhinsk, Dnepropetrovsk Region, 51900, Ukraine)

Abstract. Using the methods of physical modeling, the study of the modes of expiration of vortex gas streams of the nozzles of different dimensions for different gas flow has been carried out.

Keywords: converter, lining life, slag blowing, slag skull, vortex flow.

\section{REFERENCES}

1. Mokrinskii A.V., Lavrik A.N., Sokolov V.V., Protopopov E.V. Direction of increasing the resistance of converters' lining. Novye ogneupory. 2004, no. 8, pp. 16-18. (In Russ.).

2. Aizatulov R.S., Protopopov E.V., Sokolov V.V. etc. Sposob naneseniya shlakovogo garnisazha na futerovku konvertera [Application method for slag skull in converter lining]. Patent RF no. 2128714, 1999. (In Russ.).
3. Bagryantsev V.I., Nikolaev A.L., Poltoratskii L.M., Chevalkov A.V. Steel in Translation. 1988. Vol. 18, no. 2, pp. 59-61.

4. Bagryantsev V.I., Nikolaev A.L., Pavlenko Z.Ya. The dynamics of particles in guniting in a twisted flow. Refractories and Industrial Ceramics. 1988. Vol. 29, no. 5-6, pp. 387-391.

5. Protopopov E.V., Kalimullin R.F., Chernyatevich A.G., Kharlashin P.S. Simulation parameters of a supersonic jet in the process of converter slag blowing. Izvestiya VUZov. Chernaya metallurgiya $=$ Izvestiya - Ferrous Metallurgy. 2012, no. 10, pp. 38-43. (In Russ.).

6. Kalimullin R.F., Protopopov E.V., Chernyatevich A.G., Zhibinova I.A. Study of laws and distribution of forming gunning torch in the «hot» relining converter masses. Vestnik SibGIU. 2013, no. 3, pp. 9-13. (In Russ.).

7. Gol'dshtik M.A. Vikhrevye potoki (Eddy currents). Novosibirsk: Nauka, 1981. 367 p.

8. Gupta A., Lilley D., Syred N. Swirl flows. Abacus Press, 1984, 475 p. (Russ.ed.: Gupta A., Lilley D., Syred N. Zakruchennye potoki. Novosibirsk: Nauka, 1981. 367 p.).

9. Kutateladze S.S., Volchkov E.P., Terekhov V.I. Aerodinamika i teplomassoobmen $v$ ogranichennykh vikhrevykh potokakh [Aerodynamics and heat transfer in confined vortex flows]. Novosibirsk: AN SSSR, 1987. 282 p. (In Russ.).

Acknowledgements. Work was performed by SibGIU for the state order of the Ministry of Education of the Russian Federation, the project 2556. Received June 27, 2014 